\title{
First description of a double heterozygosity for $B R C A 1$ and $B R C A 2$ pathogenic variants in a French metastatic breast cancer patient: A case report
}

\author{
GUILLAUME MEYNARD ${ }^{1,2}$, LAURA MANSI ${ }^{1-3}$, PIERRE LEBAHAR ${ }^{4}$, CRISTIAN VILLANUEVA $^{1,2}$, \\ ELODIE KLAJER $^{1,2}$, FABIEN CALCAGNO ${ }^{1-3}$, ADRIAN VILALTA $^{4}$, MARIE CHAIX $^{5}$, \\ MARIE-AGNÈS COLLONGE-RAME ${ }^{2,6}$, CÉLINE POPULAIRE ${ }^{2,6}$, MARIE-PAULE ALGROS $^{2,7}$, \\ PRUDENCE COLPART $^{2,7}$, JULIE NEIDICH $^{4}$, XAVIER PIVOT $^{1-3}$ and ELSACURTIT ${ }^{1-3}$ \\ ${ }^{1}$ Department of Medical Oncology, University Hospital, 25000 Besançon; ${ }^{2}$ University of Franche-Comté, \\ 25000 Besançon; ${ }^{3}$ INSERM U1098, 25000 Besançon, France; ${ }^{4}$ Pathway Genomics Corporation, \\ San Diego, CA 92121, USA; ${ }^{5}$ Department of Medical Oncology, University Hospital, 21079 Dijon; \\ ${ }^{6}$ Department of Genetic Counseling, Saint Jacques Hospital, 25030 Besançon; ${ }^{7}$ Department of \\ Anatomopathology Laboratories, University Hospital, 25000 Besançon, France
}

Received August 17, 2016; Accepted January 2, 2017

DOI: $10.3892 /$ or.2017.5422

\begin{abstract}
Hereditary breast and ovarian cancer syndrome is an autosomal dominant disease caused primarily by germline mutations in the BRCA1 or BRCA2 gene. Rare cases of double heterozygosity for $B R C A 1$ and $B R C A 2$ mutations have been reported quite exceptionally in non-Ashkenazi individuals. We describe the case of a woman who developed a bilateral breast cancer, discovered concomitantly, at 46 years old. Biopsies confirmed the presence of two breast cancers with distinct histology. BRCA analysis was tested due to a positive family history of breast cancer, and two pathogenic monoallelic mutations were detected, one in the BRCAl gene and one in the $B R C A 2$ gene. There is no known Ashkenazi Jewish ancestry. We report the first description of a never described double heterozygosity for $B R C A 1$ and $B R C A 2$ pathogenic variants in a French metastatic breast cancer patient, with two distinct histology, and two distinct mutations.
\end{abstract}

\section{Introduction}

A small proportion of breast and ovarian cancer is explained by a mutation in BRCAI and BRCA2. Mutations in the BRCAI

Correspondence to: Dr Guillaume Meynard, Service d'Oncologie Médicale, Hôpital Jean Minjoz, 3 Boulevard Fleming, 25000 Besançon, France

E-mail: guillaumeynard@gmail.com

Abbreviations: BRCA gene, breast cancer gene; HER2, human epidermal growth factor receptor 2; RECIST, response evaluation criteria in solid tumors

Key words: hereditary breast and ovarian syndrome, BRCA, breast cancer or $B R C A 2$ genes, respectively located on chromosome $17 \mathrm{q} 21$ and chromosome 13q12-13, are responsible for hereditary breast and ovarian cancer syndrome (1). In France, causal mutations in $B R C A 1$ and $B R C A 2$ genes account for about $10 \%$ of this syndrome $(2,3)$. BRCAl (breast cancer 1 ) gene encodes a multifunctional protein that interacts with tumor suppressors, DNA repair proteins, cell cycle regulators, RNA polymerase II holoenzyme, transcription factors, co-repressors, chromatin remodeling enzymes, and RNA processing factors (4). BRCA1 protein, therefore, has a critical role in maintaining genomic stability with key functions in DNA repair, cell cycle progression, transcriptional regulation and ubiquitylation (4,5). BRCA2 (breast cancer 2) gene encodes a protein with rather similar roles in the DNA damage response and DNA repair pathways (5). The $B R C A 2$ protein is a tumor suppressor that mediates recruitment of the RAD51 recombinase protein to DNA double-stand breaks (5). The primary function of the $B R C A 2$ protein is to facilitate homologous recombination, an important DNA repair mechanism to maintain genomic integrity $(5,6)$. Loss or inactivation of one copy of BRCA1 or BRCA2 results in accumulation of mutations and structural changes in the genome, thereby increasing risk of cancer (6). BRCA1 and BRCA2 mutations are inherited in an autosomal dominant condition with variable penetrance (7).

The present study reports the case of a French patient with two synchronous different breast cancers metastatic at presentation who was found to carry a double, never described mutation, in BRCA1 and BRCA2 genes.

\section{Case report}

Clinical history. In July 2014, a 46-year-old woman was referred to our medical oncology unit because she had noted at self-palpation a mass in her left breast. Physical examination 


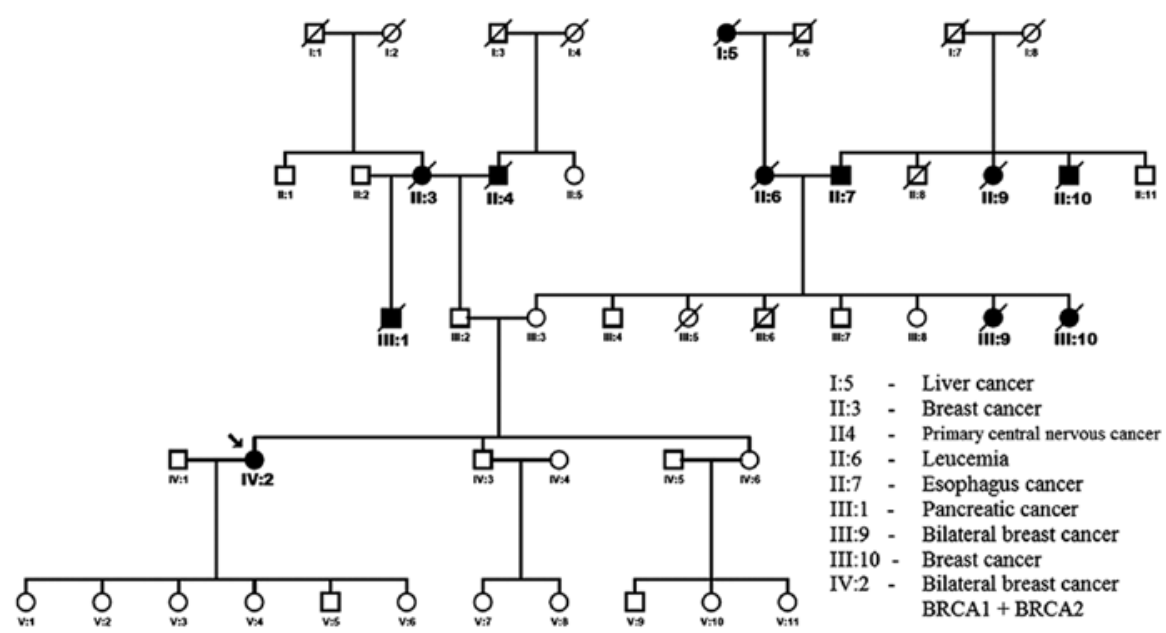

Figure 1. Family tree of the patient (indicated by the arrow). BRCA (breast cancer) analysis was tested due to a positive family history of breast cancer (II:3, III:9, III:10). Two pathogenic monoallelic mutations were detected in the proband (IV:2), one in the BRCA1 gene and one in the BRCA2 gene. There is no known Ashkenazi Jewish ancestry. Subsequent predictive testing was offered to the parents. The patient's mother (III:3) present a pathogenic monoallelic mutation in the BRCA1 gene.
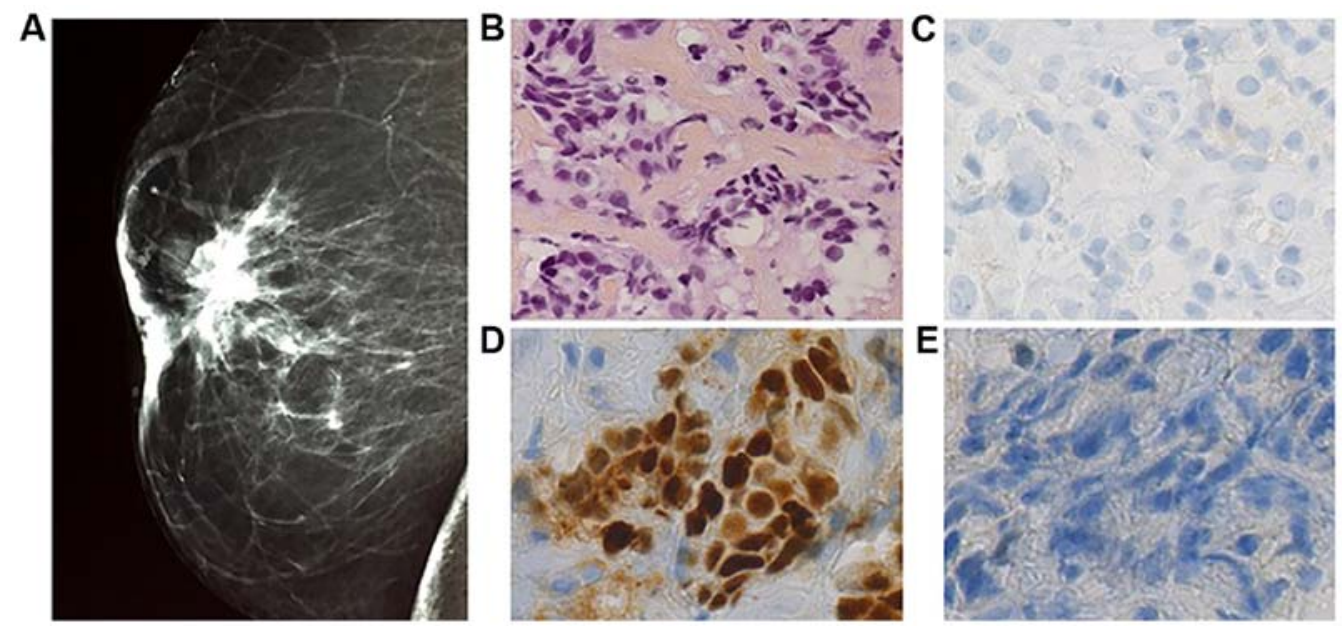

Figure 2. Right breast details, mammogram, histological and immunohistochemical studies. Medial-lateral oblique mammogram of the right breast demonstrating supra areolar retractile opacity measuring $2.2 \mathrm{~cm}$ (A). The right breast biopsy diagnosed infiltrating ductal carcinoma (B, HES staining). Elston and Ellis grade was scored at II (3.2.1). On immunohistochemical stainings, the tumor strongly expressed estrogen receptor (D), with no expression of progesterone receptor (E) and no overexpression of HER 2 (C).
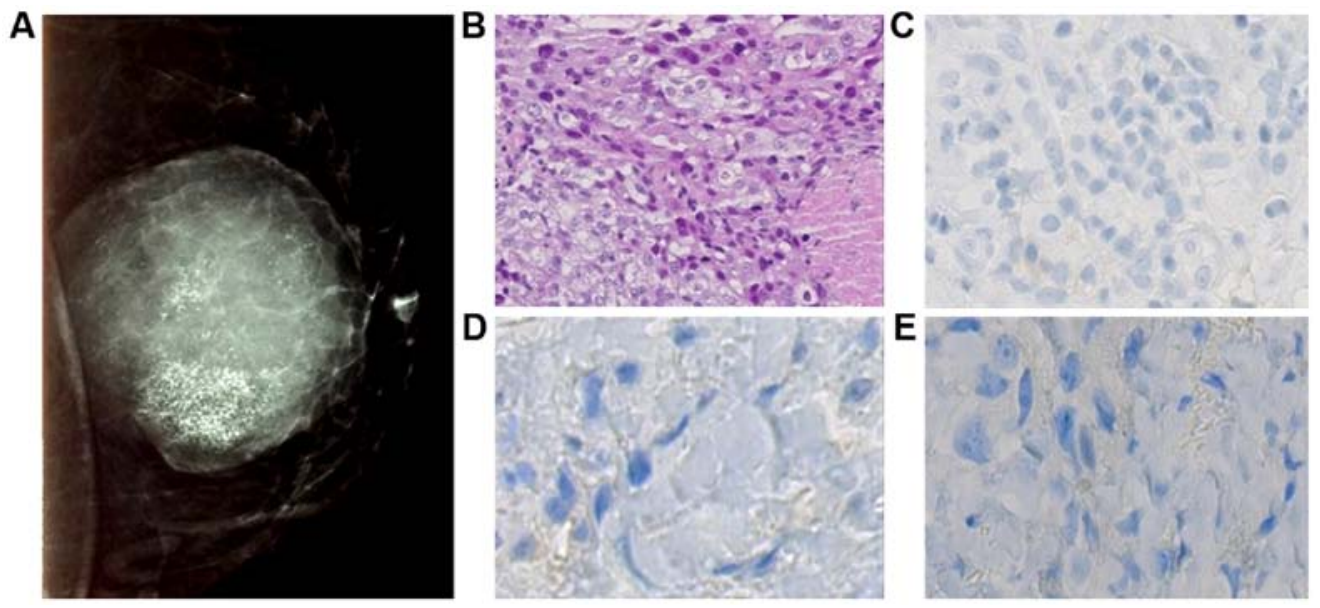

Figure 3. Left breast details, mammogram, histological and immunohistochemical studies. Medial-lateral oblique mammogram of the left breast demonstrating a large opacity in the supero-internal quadrant, measuring $10 \mathrm{~cm}$, well limited with diffuse micro-calcifications (A). Biopsies of the left breast revealed triple-negative infiltrating ductal carcinoma (B, HES staining), no overexpression of HER2 (C), no expression of estrogen receptor (D), and progesterone receptor (E). Elston and Ellis grade was scored at III (3.3.3). 


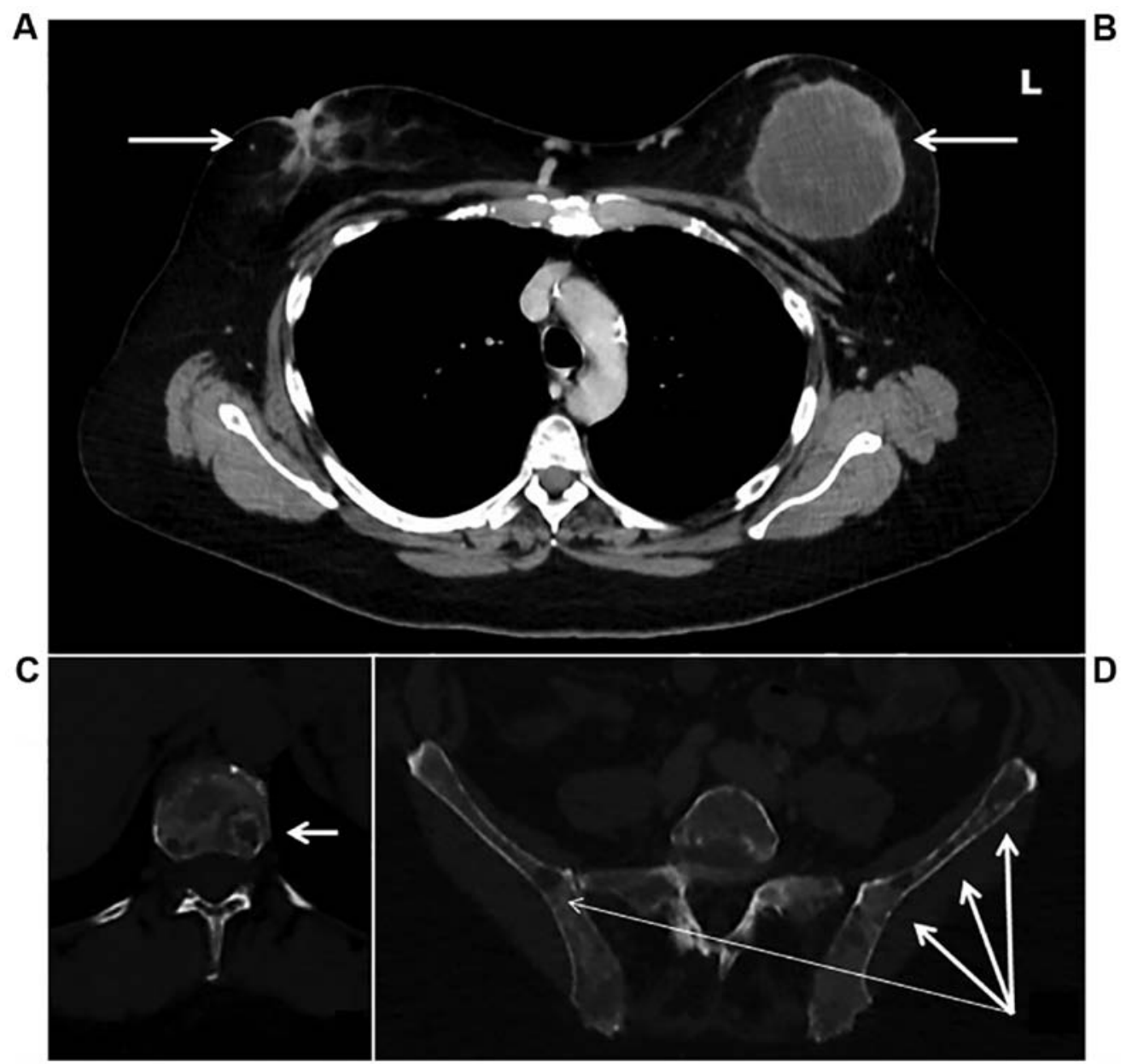

Figure 4. Initial computed tomography. (L, left side). Chest computed tomography scan axial view showing the right breast tumor measuring $2.2 \mathrm{~cm}$ with cutaneous retraction (A), and the left breast tumor measuring $10 \mathrm{~cm}$ (B). Bone window showing vertebral bone metastasis (C) and sacral bone metastasis (D).

confirmed an inflammatory indurated mass of the left breast measuring $12 \mathrm{~cm}$. Moreover, it revealed an areola retraction of the right breast measuring $3 \mathrm{~cm}$ without any discharge. There were bilateral clinically fixed axillary lymph nodes.

The proband has a positive familial history of breast carcinomas and other cancers (Fig. 1). Her paternal grandmother (II:3) died at the age of 72 years from breast cancer. One of her maternal aunts (III:9) developed bilateral breast cancer at 40 years and died at 59 years. Another maternal aunts (III:10) developed breast cancer at 45 years and died at 66 years. Her father's half-brother died from a pancreatic adenocarcinoma (III:1).

We noted in her personal medical history, cholecystectomy and active tobacco intoxication measured at 21 pack-years. Initially she had no regular treatment. The patient started her first period at the age of 14 . She had 6 healthy children and she breast-fed two of them. There were no abortion rating, no miscarriages. After the birth of her sixth child, a hormonal intrauterine device was inserted and then removed in July 2014 at breast cancer diagnosis. Chemotherapy induced a stop of menses.

Bilateral digital mammography was performed. Right breast was Bi Rads type II classified, with supra areolar retractile opacity measuring $2.2 \mathrm{~cm}$ without any calcification (Fig. 2). In left breast, opacity in the supero-internal quadrant, measuring $10 \mathrm{~cm}$, well limited with diffuse microcalcifications was observed (Fig. 3).

Biopsies confirmed the presence of two breast cancers with distinct histology. The right breast biopsy diagnosed infiltrating ductal carcinoma of no special type. Elston and Ellis grade was scored at II (3.2.1). On immunohistochemical staining, the tumor strongly expressed estrogen receptor (95\%), with no expression of progesterone receptor and no overexpression of HER 2. Ki 67 expression was assessed at 14\% (Fig. 2). It was T4bN2 classified.

The left breast biopsy diagnosed a triple negative infiltrating ductal carcinoma of no special type (no expression of estrogen receptor, progesterone receptor and HER2). Elston and Ellis grade was scored at III (3.3.3) and cytokeratin 5 and 6 were expressed in $40 \%$ of cells (Fig. 3). It was T4dN2 classified.

A staging computed tomography and an isotopic bone scan detected diffuse bone lesions located on the entire spine, pelvis, skull, and sternum without any visceral metastatic lesions (Fig. 4). CA 15.3 marker was measured at $2678 \mathrm{U} / \mathrm{ml}$. Computed tomography and pelvic ultra sound did not detect ovarian lesion.

Gene testing. The patient was tested for germline mutations in $B R C A 1$ and $B R C A 2$ genes after written informed and signed 
A BRCA1
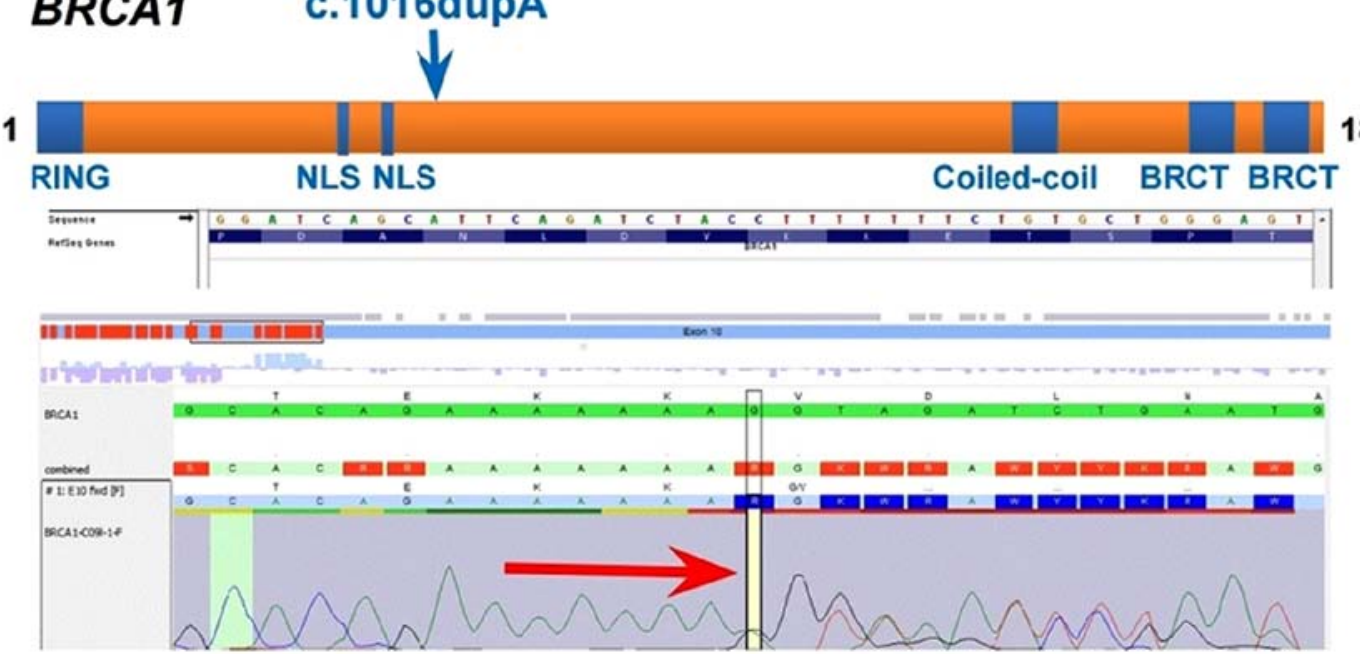

B BRCA2

1

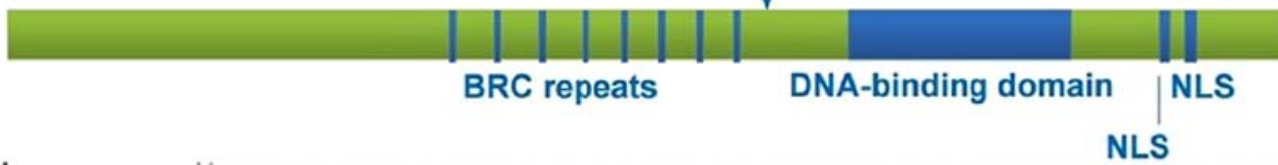

c.6814delA

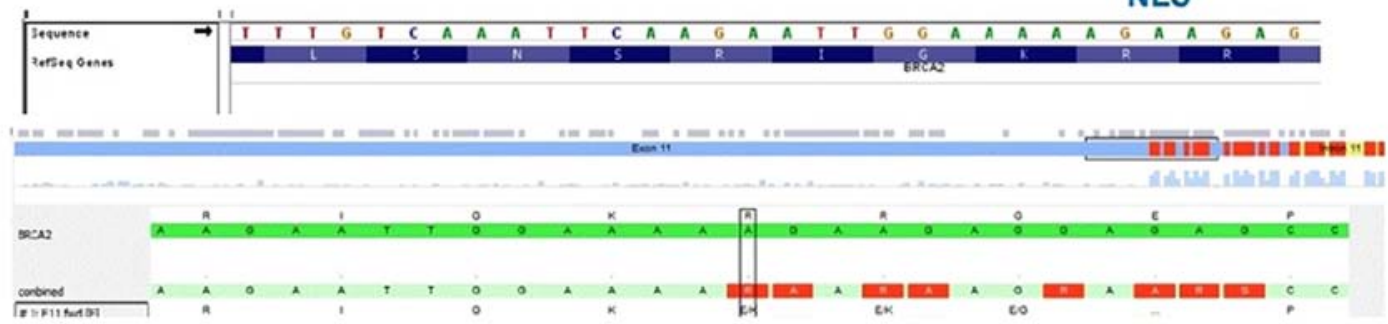

Figure 5. Identification of the $B R C A 1$ and $B R C A 2$ pathogenic variants by NGS and Sanger sequencing. Schematic diagrams show the approximate locations (blue arrows) of the BRCA1 c.1016dupA (p.V340GfsX6) (A) and BRCA2 c.6814delA (p.R2272EfsX8) (B) pathogenic variants identified in this patient. Red arrows indicate the DNA sequence that varied from the reference sequence.

consent. Written informed consent was obtained from the patient and her parents for publication of this case report and any accompanying images. Ethics approval was not applicable. The BRCA True ${ }^{\mathrm{TM}}$ test (Pathway Genomics Laboratories) is designed to analyze the coding and flanking regions of BRCA1 and BRCA2 genes associated with hereditary breast and ovarian cancer by next-generation sequencing-base (NGS) and Sanger sequencing. Genomic DNA (gDNA) is extracted from the patient's specimen (peripheral blood and saliva sample), and evaluated for quality and quantity using standard procedures. The gDNA is processed to enrich for the targeted exons and flanking regions in a PCR-based reaction with target-specific primers. Massive parallel sequencing is carried out on the enriched target DNA to detect variants in these regions. Sanger DNA sequencing is utilized for targeted gene regions that are insufficiently covered on NGS for variant detection and to confirm specific findings when suspected pathogenic or novel variants are detected. Gross deletions and duplications in BRCAI and BRCA2 genes are identified by multiplex ligation-dependent probe amplification. Two pathogenic monoallelic mutations were detected, one in each gene: c.1016dupA (p.V340Gfs*6) mutation in the BRCAl gene and c.6814delA (p.R2272Efs*8) mutation in the BRCA2 gene
(Fig. 5). The BRCAl c.1016dupA (p.V340Gfs*6) pathogenic variant, also known as 1135insA or 1135dup, is predicted to truncate the $B R C A 1$ protein. The $B R C A 2$ c.6814delA (p.R2272Efs*8) pathogenic variant, also known as 7042delA, is predicted to truncate the $B R C A 2$ protein. Mutation designation is according to the American College of Medical Genetics (ACMG) guidelines.

Genetic management. After receiving these results, the proband was referred to genetic counseling. Subsequent predictive testing was offered to the proband's parents. The proband's asymptomatic mother (Fig. 1, indicated by III:3) was found to be positive for the same mutation in the $B R C A l$ gene. She is 69 years old. Bilateral digital mammography and pelvic ultrasound was performed. To date she has had no cancer, and has now entered into a specific follow-up program. The proband's father (Fig. 1, indicated by III:2), aged 68 did not carry either mutation. He has had no cancer.

Patient management and outcomes. The patient received a first line of chemotherapy by weekly paclitaxel $\left(90 \mathrm{mg} / \mathrm{m}^{2}\right)$ with bevacizumab every 2 weeks $(10 \mathrm{mg} / \mathrm{kg})$ for 6 months. Treatment was well tolerated and associated with monthly 
injections of denosumab $120 \mathrm{mg}$. After 6 months of treatment, a good partial response according to RECIST criteria 1.1 was observed with a decrease of the two breast lesions (from $93 \mathrm{~mm}$ to $57 \mathrm{~mm}$ in the left breast and from 24 to $13 \mathrm{~mm}$ in the right breast). Axillary lymph nodes were not any more palpable. Computed tomography and bone scan revealed an improvement on bone lesions associated with a decrease of CA 15.3 at $285 \mathrm{U} / \mathrm{ml}$. We decided to focus on systemic therapy, and to delay the two primary breast cancer surgery. A maintenance therapy with capecitabine $2000 \mathrm{mg}$ twice a day and bevacizumab $15 \mathrm{mg} / \mathrm{kg}$ each 21 days was started (8). Twenty months later, the patient is still alive with no criteria of progression of the disease; she has recovered her regular activities and comes every 3 weeks for a maintenance treatment and follow-up.

\section{Discussion}

To the best of our knowledge, we described the first case of a double heterozygosity for $B R C A 1$ and $B R C A 2$ pathogenic variants in a French metastatic breast cancer patient, with mutation c.1016dupA in BRCAl and mutation c.6814delA in $B R C A 2$ gene never described together so far. The co-existence in an individual of mutations in the BRCAI and $B R C A 2$ genes is a very rare finding, particularly in nonAshkenazi individuals $(1,9)$, and our patient has no known Ashkenazi heritage.

Moreover, we determined that the patient inherited the BRCA1 mutation from her mother. Regarding the BRCA2 mutation, it could be a de novo mutation or maybe the father is not the biological father. Ethical French rules do not allow us to genetically test this last hypothesis.

Leegte et al presented a review of the literature and described the phenotypic expression of double heterozygosis for BRCA1 and BRCA2 in 34 women (9). All cases presented an Ashkenazi mutation. The highest risk of cancer appears to occur in a combination of 5382insC and 6174delT for $B R C A 1$ and BRAC2 genes, respectively $(1,9,10)$. The population carrier frequency of 185delAG, 5382insC (BRCA1), and 6174delT (BRCA2) is estimated to be $0.92,0.26$, and 1.20 , respectively, in the Ashkenazi population $(11,12)$ adding up to approximately $2.4 \%$. Peto et al estimated the prevalence of $B R C A 1$ and BRCA2 mutation carriers to be $0.11 \%$ and $0.12 \%$, respectively, in the non-Ashkenazi (UK) population, which adds up to $0.23 \%$ (13). This indicates that the chance for an occurrence of double heterozygosity in these populations is between 1 in 1,800 to 190,000 , respectively.

Furthermore, the BRCAl c.1016dupA variant is considered a Norwegian founder mutation, but has also been observed in individuals who are of French-Canadian, French, Italian or Dutch ancestry (2,14-16). The BRCA2 c.6814delA (p.R2272Efs*8) pathogenic variant, has been identified in individuals with a personal or family history of breast and/ or ovarian cancer $(17,18)$. The clinical presentation was advanced and unfavorable, it may raise up the possibility of a pejorative impact of either involved mutations or resulting from their association. Nevertheless, review of the literature suggests that the co-existence of BRCA1 and BRCA2 mutations likely not cause a more severe phenotype of breast cancer $(1,9)$.
In this patient, concomitant mutations of both $B R C A l$ and $B R C A 2$ might lead to two different breast cancers with distinct histologic features. It might suggest that even if initially all breast cells present the same genetic characteristics, oncogenesis of the two tumors are different and lead to two breast cancers of different subtypes: a triple-negative and a luminal breast cancer. The next step could be to sequence the two breast tumors to assess if cancer cells had lost both the wildtype alleles of $B R C A 1$ and $B R C A 2$. It could explain whether the oncogenesis of these tumors is driven by a loss of function of $B R C A 1$ and/or BRCA2. A comparative genomic analysis of both tumors could prove extremely informative on early and specific BRCA associated tumorigenesis mechanisms, but unfortunately, it is not available in our center.

The key-information of this case can be summarized in three points: we report a double heterozygosity with a never described association of two BRCA mutations (c.1016dupA in $B R C A 1$ and c.6814delA in BRCA2 gene). These two mutations were discovered with the diagnosis of two distinct concomitant pathological type of breast cancer. We search for BRCAl and $B R C A 2$ mutations in the parents; one mutation is inherited from the mother and the other could be de novo.

\section{References}

1. Smith M, Fawcett S, Sigalas E, Bell R, Devery S, Andrieska N and Winship I: Familial breast cancer: Double heterozygosity for BRCA1 and BRCA2 mutations with differing phenotypes. Fam Cancer 7: 119-124, 2008.

2. Caputo S, Benboudjema L, Sinilnikova O, Rouleau E, Béroud C and Lidereau R; French BRCA GGC Consortium: Description and analysis of genetic variants in French hereditary breast and ovarian cancer families recorded in the UMD-BRCA1/BRCA2 databases. Nucleic Acids Res 40: D992-D1002, 2012.

3. Oncogénétique en 2014 - Consultations, laboratoires et prise en charge - reference: APPDECONCOG15, Institut National Du Cancer. (In French).

4. Deng CX and Brodie SG: Roles of BRCA1 and its interacting proteins. BioEssays 22: 728-737, 2000.

5. Roy R, Chun J and Powell SN: BRCA1 and BRCA2: Different roles in a common pathway of genome protection. Nat Rev Cancer 12: 68-78, 2011.

6. Petrucelli N, Daly MB and Feldman GL: BRCA1 and BRCA2 hereditary breast and ovarian cancer. In: GeneReviews(®) [Internet]. Pagon RA, Adam MP, Ardinger HH, et al (eds). University of Washington, Seattle, WA, 1993-2016.

7. Thull DL and Vogel VG: Recognition and management of hereditary breast cancer syndromes. Oncologist 9: 13-24, 2004.

8. Gligorov J, Doval D, Bines J, Alba E, Cortes P, Pierga JY, Gupta V, Costa R, Srock S, de Ducla S, et al: Maintenance capecitabine and bevacizumab versus bevacizumab alone after initial first-line bevacizumab and docetaxel for patients with HER2-negative metastatic breast cancer (IMELDA): A randomised, open-label, phase 3 trial. Lancet Oncol 15: 1351-1360, 2014.

9. Leegte B, van der Hout AH, Deffenbaugh AM, Bakker MK, Mulder IM, ten Berge A, Leenders EP, Wesseling J, de Hullu J, Hoogerbrugge N, et al: Phenotypic expression of double heterozygosity for BRCA1 and BRCA2 germline mutations. J Med Genet 42: e20, 2005.

10. Choi DH, Lee MH and Haffty BG: Double heterozygotes for non-Caucasian families with mutations in BRCA-1 and BRCA-2 genes. Breast J 12: 216-220, 2006.

11. Fodor FH, Weston A, Bleiweiss IJ, McCurdy LD, Walsh MM, Tartter PI, Brower ST and Eng CM: Frequency and carrier risk associated with common BRCA1 and BRCA2 mutations in Ashkenazi Jewish breast cancer patients. Am J Hum Genet 63: 45-51, 1998.

12. Satagopan JM, Offit K, Foulkes W, Robson ME, Wacholder S, Eng CM, Karp SE and Begg CB: The lifetime risks of breast cancer in Ashkenazi Jewish carriers of BRCA1 and BRCA2 mutations. Cancer Epidemiol Biomarkers Prev 10: 467-473, 2001. 
13. Peto J, Collins N, Barfoot R, Seal S, Warren W, Rahman N, Easton DF, Evans C, Deacon J and Stratton MR: Prevalence of BRCA1 and BRCA2 gene mutations in patients with early-onset breast cancer. J Natl Cancer Inst 91: 943-949, 1999.

14. Andersen TI, Børresen AL and Møller P: A common BRCA1 mutation in Norwegian breast and ovarian cancer families? Am J Hum Genet 59: 486-487, 1996.

15. Simard J, Tonin P, Durocher F, Morgan K, Rommens J, Gingras S, Samson C, Leblanc JF, Bélanger C, Dion F, et al: Common origins of BRCA1 mutations in Canadian breast and ovarian cancer families. Nat Genet 8: 392-398, 1994.

16. Dørum A, Heimdal K, Hovig E, Inganäs $M$ and Møller P: Penetrances of BRCA1 1675delA and 1135insA with respect to breast cancer and ovarian cancer. Am J Hum Genet 65: 671-679, 1999.
17. Novaković S, Milatović M, Cerkovnik P, Stegel V, Krajc M, Hočevar M, Zgajnar J and Vakselj A: Novel BRCA1 and BRCA2 pathogenic mutations in Slovene hereditary breast and ovarian cancer families. Int J Oncol 41: 1619-1627, 2012.

18. Tea M-KM, Kroiss R, Muhr D, Fuerhauser-Rappaport C, Oefner P, Wagner TM and Singer CF: Central European BRCA2 mutation carriers: Birth cohort status correlates with onset of breast cancer. Maturitas 77: 68-72, 2014. 